\title{
Inertia effects in rheometrical flow systems Part 2: The balance rheometer
}

\author{
H. A. Waterman
}

With 4 figures

(Received October 1, 1976)

\begin{tabular}{|c|c|}
\hline \multicolumn{2}{|l|}{ Notations } \\
\hline$a$ & $\begin{array}{l}\text { distance between rotation axis of or- } \\
\text { thogonal rheometer }\end{array}$ \\
\hline$b$ & body force \\
\hline$b_{r}, b_{\theta}, b_{\phi}$ & physical components of the body force \\
\hline $\mathcal{C}$ & $\begin{array}{l}\text { velocity of propagation of shear waves } \\
\text { in a Hookean material }\end{array}$ \\
\hline$c^{*}=c^{\prime}+i c^{\prime \prime}$ & $\begin{array}{l}\text { complex velocity of propagation of } \\
\text { shear waves in a linear viscoelastic } \\
\text { fluid }\end{array}$ \\
\hline$e$ & dilatation \\
\hline$h$ & $\begin{array}{l}\text { gap distance between hemispheres of } \\
\text { balance rheometer resp. between plates } \\
\text { of orthogonal rheometer }\end{array}$ \\
\hline$i$ & $\sqrt{-1}$ \\
\hline$j_{n}(x)$ & $\begin{array}{l}\text { spherical Bessel-function of the first } \\
\text { kind and order } n\end{array}$ \\
\hline$k^{*}=k^{\prime}-i k^{\prime \prime}$ & complex wave number \\
\hline$k$ & $-(\rho \omega / 2 \eta)^{\frac{1}{2}}$ \\
\hline$k_{e}$ & wave number in a Hookean material \\
\hline$n$ & non-negative integer \\
\hline$r$ & spherical polar coordinate \\
\hline$r_{0}$ & $\begin{array}{l}\text { radius of plates of orthogonal } \\
\text { rheometer }\end{array}$ \\
\hline$r_{1}$ & $\begin{array}{l}\text { radius of inner hemisphere of balance } \\
\text { rheometer }\end{array}$ \\
\hline$r_{2}$ & $\begin{array}{l}\text { radius of outer hemisphere of balance } \\
\text { rheometer }\end{array}$ \\
\hline$t$ & time \\
\hline$u_{r}, u_{\theta}, u_{\phi}$ & physical components of displacement \\
\hline$v_{r}, v_{\theta}, v_{\phi}$ & physical components of velocity \\
\hline$x, y, z$ & cartesian coordinates \\
\hline$y_{n}(z)$ & $\begin{array}{l}\text { spherical Bessel-function of the second } \\
\text { kind and order } n\end{array}$ \\
\hline$A, B, C$ & integration constants \\
\hline$F$ & force \\
\hline$G^{*}=G^{\prime}+i G^{\prime \prime}$ & complex shear modulus \\
\hline$J_{\frac{1}{2}}(z)$ & $\begin{array}{l}\text { Bessel-function of the first kind and } \\
\text { order } \frac{1}{2}\end{array}$ \\
\hline$M$ & couple \\
\hline$P_{v}^{\mu}(x)$ & $\begin{array}{l}\text { associated Legendre-function (spher- } \\
\text { ical harmonic) of the first kind, degree } v \\
\text { and order } \mu\end{array}$ \\
\hline $\begin{array}{l}P_{n}(x) \\
O^{\mu}(x)\end{array}$ & Legendre polynomial of degree $n$ \\
\hline$Q_{v}^{\mu}(x)$ & associated Legendre-function (spher- \\
\hline
\end{tabular}

$S$ $Y_{\frac{1}{2}}(z)$ $\delta$ $\varepsilon$ $\eta$ $\eta$ $\hat{\lambda}$ A $\rho$ $\tau$ $\phi$ spherical polar coordinate (1) angular velocity $\omega_{r}, \omega_{\theta}, \omega_{\phi} \quad$ physical components of the spin tensor

\section{Introduction}

In a previous paper (1) a simple procedure was introduced to calculate the flow field of a linear viscoelastic fluid in the orthogonal rheometer when fluid inertia is taken into account. This procedure is basically founded on the concept that in a coordinate system fixed to one of the plates the deformation of the sample can be described as a superposition of two harmonic plane shear deformations, polarized perpendicular to each other, having the same amplitude but showing a phase difference of $\pi / 2$ radians. Inertia effects manifest itself in a finite velocity of propagation of the occurring plane shear waves. The forces on the plates, too, are readily found with this procedure.

In the present paper the same procedure will be applied to the balance rheometer. Here, in a rotating coordinate system, the deformation of the sample can also be described as a superposition of two harmonic shear deformations. A straightforward calculation leads to an exact 
solution of the flow field of a linear viscoelastic fluid from which exact solutions for the forces and the couples on the hemispheres are deduced.

In an earlier paper Walters (2) presented an expression for the couples in the form of series expansions. Extension of this work by Bowen (3) led to series expansions for the forces. It will be shown that neither the series expansions given by Walters (2) nor the series expansions given by Bowen (3) are in accordance with series expansions of the exact solutions.

\section{Flow field in the balance rheometer}

\subsection{Flow field of a linear viscoelastic fluid neglecting inertia effects}

Fig. 1a shows the geometry of the balance rheometer. The fluid is contained between two concentric hemispheres. In operation the inner hemisphere is rotated clockwise with constant angular velocity $\omega$ about a vertical axis. The outer hemisphere, the axis of which is supposed to have a frictionless bearing, rotates with the same angular velocity about an axis which makes a small angle $\varepsilon$ with the vertical, see fig. $1 \mathrm{~b}$. It is clear that for $\varepsilon=0$ the fluid performs a rigid rotation. Now suppose that the rheometer is filled when $\varepsilon=0$ and consider material lines passing through the common centre of both hemispheres. Subsequently the vertical axis of the outer hemisphere is tilted over the angle $\varepsilon$ in the $x-z$ plane and set into rotation with angular velocity $\omega$. In a rotating coordinate system fixed to a particle on the inner hemisphere particles situated on the material line passing through this particle will turn ellipses with respect to this particle. Likewise two arbitrary particles on this material line will, in this coordinate system, turn ellipses with respect to each other. It is to be noted that these ellipses are turned anti-clockwise with angular velocity $\omega$. In particular for particles located on the material line which originally coincided with the $z$-axis these ellipses are circles, while for particles located on the line originally coincident with the $x$-axis the ellipses degenerate into straight lines, see fig. $1 \mathrm{~b}$. So we find that the deformation of the sample, although it is inhomogeneous, is locally harmonic. We can therefore make use of the correspondence principle, which states a.e. that for each problem in linear viscoelasticity for which the deformations are harmonic, the solution can be found by solving the analogous problem with the same boundary conditions for a linear elastic material and substituting the complex moduli in the final relations.

It is worth to note that the harmonicity of the deformation is ultimately due to the fact that in a rotating coordinate system fixed to the inner hemisphere and oriented as shown in fig. $1 \mathrm{a}$ the sample is subjected to two harmonic oscillations, one about the $x$-axis and the other about the $y$ axis, both with the angle amplitude $\varepsilon$ but showing a phase difference of $\pi / 2$ radians. The formal prove of this statement, which holds for small values of $\varepsilon$, is readily given.

As suggested by the geometry we will use spherical polar coordinates $r, \theta$ and $\phi$, defined by

$$
\left.\begin{array}{l}
x=r \sin \theta \cos \phi \\
y=r \sin \theta \sin \phi \\
z=r \cos \theta
\end{array}\right\}
$$

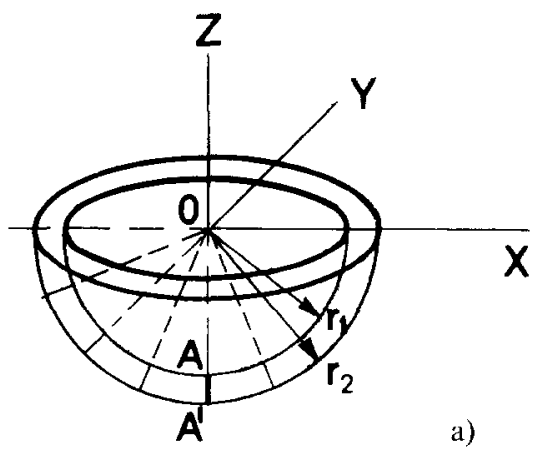

a)

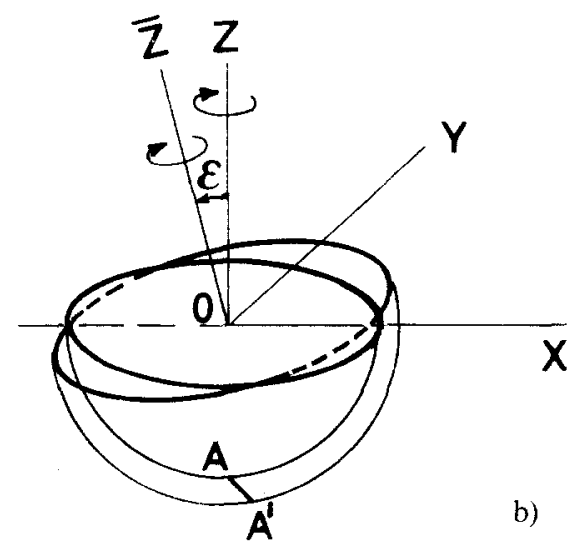

Fig. 1. Geometry of the balance rheometer.

a) Balance rheometer with the sample in the initial (undeformed) state; b) Balance rheometer during measurements 
We also introduce a further set of coordinates $(\bar{r}, \bar{\theta}, \bar{\phi})$ related to the $0 \bar{z}$ and $0 y$ axes. The following relations hold:

$\cos \bar{\theta}=\cos \theta \cos \varepsilon+\sin \theta \cos \phi \sin \varepsilon$ $\sin \bar{\theta} \cos \phi=\sin \theta \cos \phi \cos \varepsilon-\cos \theta \sin \varepsilon\}$.

The boundary conditions for the outer hemisphere are:

$v_{\bar{r}}=0 ; v_{\bar{\theta}}=0 ; v_{\bar{\phi}}=\omega r_{2} \sin \bar{\theta} \quad$ on $\quad r=r_{2}$.

A transformation of coordinates and use of [2] yields, in place of [3]:

$v_{r}=0 ; v_{\theta}=-\varepsilon \omega r_{2} \sin \phi ;$

$v_{\phi}=\omega r_{2}[\sin \theta-\varepsilon \cos \theta \cos \phi]$ on $r=r_{2}$.

In [4] second order terms in $\varepsilon$ have been ignored. From [4] it follows for the velocity of a particle on the outer sphere with respect to a rotating coordinate system fixed to the inner sphere:

$v_{r}^{\prime}=0 ; v_{\theta}^{\prime}=-\varepsilon \omega r_{2} \sin \phi ;$

$v_{\phi}^{\prime}=-\varepsilon \omega r_{2} \cos \theta \cos \phi$.

With $\phi=\phi_{0}+\omega t$ the displacements are found from [5] to be:

$u_{r}=0 ; u_{\theta}=\varepsilon r_{2} \cos \omega t$

$u_{\phi}=-\varepsilon r_{2} \cos \theta \sin \omega t$,

where the integration constants satisfy the condition that the experiment starts at $t=0$.

Eliminating $t$ from [6] leads to the equation of the orbit of a particle:

$\frac{u_{\theta}^{2}}{\varepsilon^{2} r_{2}^{2}}+\frac{u_{\phi}^{2}}{\varepsilon^{2} r_{2}^{2} \cos ^{2} \theta}=1$.

Now consider a rotation of the outer hemisphere about the $y$-axis over the angle $\varepsilon(t)$. Again ignoring second order terms in $\varepsilon(t)$ it follows from [2]:

$\left.\begin{array}{rl}d \theta & =-\varepsilon(t) \cos \phi \\ d \phi & =\varepsilon(t) \operatorname{ctg} \theta \sin \phi\end{array}\right\}$.

So with $\varepsilon(t)=\varepsilon \sin \omega t$ :

$\left.\begin{array}{l}u_{1 \theta}=-\varepsilon r_{2} \cos \phi \sin \omega t \\ u_{1 \phi}=\varepsilon r_{2} \cos \theta \sin \phi \sin \omega t\end{array}\right\}$.

Likewise for a rotation of the outer hemisphere over the angle $\varepsilon(t)$ about the $x$-axis it is found: $\left.\begin{array}{l}\cos \bar{\theta}=\sin \theta \sin \phi \sin \varepsilon(t)+\cos \theta \cos \varepsilon(t) \\ \sin \bar{\theta} \sin \bar{\phi}=\sin \theta \sin \phi \cos \varepsilon(t)-\cos \theta \sin \varepsilon(t)\end{array}\right\}$.

Again ignoring second order terms in $\varepsilon(t)$ we find from [10]:

$\left.\begin{array}{rl}d \theta & =-\varepsilon(t) \sin \phi \\ d \phi & =-\varepsilon(t) \operatorname{ctg} \theta \cos \phi\end{array}\right\}$

from which it follows with $\varepsilon(t)=\varepsilon \cos \omega t$ :

$\left.\begin{array}{l}u_{2 \theta}=-\varepsilon r_{2} \sin \phi \cos \omega t \\ u_{2 \phi}=-\varepsilon r_{2} \cos \theta \cos \phi \cos \omega t\end{array}\right\}$.

From [9] and [12] it is found:

$$
\begin{aligned}
u_{\phi}^{2}+\cos ^{2} \theta u_{\theta}^{2} & =\left(u_{1 \phi}+u_{2 \phi}\right)^{2}+\cos ^{2} \theta\left(u_{1 \theta}+u_{2 \theta}\right)^{2} \\
& =\varepsilon^{2} r_{2}^{2} \cos ^{2} \theta,
\end{aligned}
$$

which is identical with [7] and proves our statement.

There is one special material line in the set chosen, i.e. the line joining the south poles of the hemispheres when $\varepsilon=0$. It is evident that with the instrument in operation this line is fixed in space. Surfaces with $r=$ constant will rotate rigidly with angular velocity $\omega$ about its point of intersection with this material line. As in the rotating coordinate system this line describes a solid of revolution, see fig. $1 \mathrm{~b}$, it is clear that the position of a point of this line with respect to the $z$-axis is determined by the amplitude of one of the composing harmonic oscillations at the same $z$-coordinate.

When inertia effects are neglected the problem is reduced to the determination of the equation of the material line $A-A^{\prime}$ in fig. $1 \mathrm{~b}$ after tilting the outer hemisphere over the angle $\varepsilon$ in the $x-z$ plane. (Gravitational forces are neglected throughout in this paper.) Moreover it should be noticed that now the Navier equation for an incompressible linear elastic solid renders into the Navier Stokes equation for an incompressible Newtonian liquid when the displacements are substituted by the velocities. Now the flow field of a Newtonian fluid contained between two concentric spheres, the outer of which rotates with the angular velocity $\omega$ about the $z$ axis is, when inertia effects are neglected, well-known (4) and reads:

$v_{r}=0 ; v_{\theta}=0 ; v_{\phi}=\omega r \sin \theta\left(r-\frac{r_{1}^{3}}{r^{2}}\right)$, 
where

$\gamma=r_{2}^{3} /\left(r_{2}^{3}-r_{1}^{3}\right)$.

So for the displacements in our case we find:

$u_{r}=0 ; u_{\theta}=0 ; u_{\phi}=\varepsilon \gamma \sin \theta\left(r-\frac{r_{1}^{3}}{r^{2}}\right)$.

The same applies to a linear viscoelastic fluid. Surfaces with $r=c$ therefore rotate rigidly about the point

$r=c ; \theta=\pi / 2 ; \phi=\varepsilon \gamma\left\{r_{0}-\left(r_{1}^{3} / r_{0}^{2}\right)\right\}$.

\subsection{Flow field of a linear viscoelastic fluid including inertia effects}

In accordance with earlier investigations of the balance rheometer $(2,5)$ edge effects will be ignored. Further we assume that the fluid is incompressible, so that centripetal forces do not effect the flow field but merely appear in the pressure term. So we can safely work in a rotating coordinate system. The influence of the inertia is confined to the velocity of the shear waves in the sample, which now will be finite. The problem of finding the locus of the points about which rigid rotation of surfaces with $r$ is constant takes place therefore reduces to finding the displacement amplitude in the sample for $\theta=\pi / 2$ taking wave propagation into account.

\section{Calculation of the flow field including inertia effects}

\subsection{Flow field of a Newtonian fluid}

In spherical polar coordinates the displacement equations of motion for an isotropic linear elastic material read:

$$
\begin{gathered}
(\Lambda+2 G) \frac{\partial e}{\partial r}-\frac{2 G}{r \sin \theta} \frac{\partial}{\partial \theta}\left(\omega_{\phi} \sin \theta\right) \\
+\frac{2 G}{r \sin \theta} \frac{\partial \omega_{\theta}}{\partial \phi}+\rho b_{r}=\rho \frac{\partial^{2} u_{r}}{\partial t^{2}} \\
\frac{(\Lambda+2 G)}{2} \frac{\partial e}{\partial \theta}-\frac{2 G}{r \sin \theta} \frac{\partial \omega_{r}}{\partial \phi}+\frac{2 G}{r \sin \theta} \frac{\partial}{\partial r} \\
\frac{A+2 G}{r \sin \theta} \frac{\partial e}{\partial \phi}-\frac{2 G}{r} \frac{\partial}{\partial r}\left(r \omega_{\phi} \sin \theta\right)+\rho b_{\theta}=\rho \frac{\partial^{2} u_{\theta}}{\partial t^{2}} \\
+\rho b_{\phi}=\rho \frac{\partial^{2} u_{\phi}}{\partial t^{2}} \frac{\partial \omega_{r}}{\partial \theta}
\end{gathered}
$$

where

$$
\begin{aligned}
e= & \frac{1}{r^{2} \sin \theta}\left[\frac{\partial}{\partial r}\left(r^{2} u_{r} \sin \theta\right)\right. \\
& \left.+\frac{\partial}{\partial \theta}\left(r u_{\theta} \sin \theta\right)+\frac{\partial}{\partial \phi}\left(r u_{\theta}\right)\right]
\end{aligned}
$$

and

$$
\begin{array}{ll}
\omega_{r}=\frac{1}{2}\left[\frac{1}{r \sin \theta} \frac{\partial}{\partial \theta}\left(u_{\phi} \sin \theta\right)-\frac{1}{r \sin \theta} \frac{\partial u_{\theta}}{\partial \phi}\right] \\
\omega_{\theta}=\frac{1}{2}\left[\frac{1}{r \sin \theta} \frac{\partial u_{r}}{\partial \phi}-\frac{1}{r} \frac{\partial\left(r u_{\phi}\right)}{\partial r}\right] \\
\omega_{\phi}=\frac{1}{2}\left[\frac{1}{r} \frac{\partial}{\partial r}\left(r u_{\theta}\right)-\frac{1}{r} \frac{\partial u_{r}}{\partial \theta}\right] .
\end{array}
$$

$\Lambda$ and $G$ are the second and first Lamé functions respectively, $b$ the body force and $\rho$ the density.

As we have to determine the displacement amplitude due to an oscillation of the outer hemisphere about a horizontal axis it is of some convenience to change the orientation of the Cartesian coordinate system as shown in fig. 2 and to consider an oscillation about the new $z$-axis.

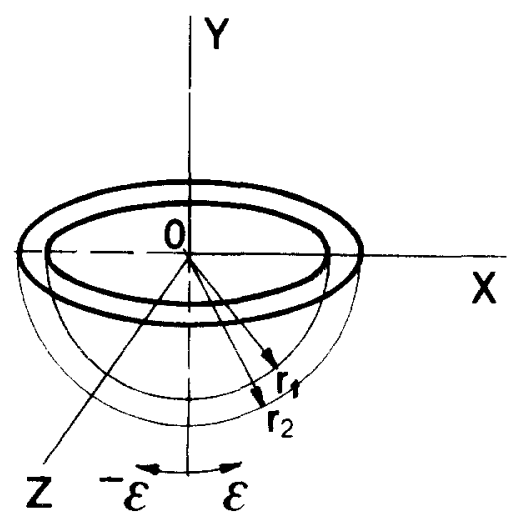

Fig. 2. Illustration to the calculation of the flow field

In accordance with [3] we then have:

$$
\begin{aligned}
& u_{r}=0 \\
& u_{0}=0
\end{aligned}
$$

while for symmetry reasons:

$\frac{\partial u_{\phi}}{\partial \phi}=0$

$b=0$. 
The boundary conditions are:

$u_{\phi}=r_{2} \varepsilon \sin \theta \exp i \omega t$ for $r=r_{2}$

$u_{\phi}=0$

for $r=r_{1}$.

From [18] and [19] it is seen that the first two equations of [16] are satisfied automatically. Moreover we find $e=0$, i. e. no volume dilatation takes place. So we are left with the last equation of [16], which takes the form:

$\frac{\partial^{2} u_{\phi}}{\partial r^{2}}+\frac{2}{r} \frac{\partial u_{\phi}}{\partial r}+\frac{1}{r^{2}} \frac{\partial^{2} u_{\phi}}{\partial \theta^{2}}+\frac{\operatorname{ctg} \theta}{r^{2}} \frac{\partial u_{\phi}}{\partial \theta}$

$$
-\frac{u_{\phi}}{r^{2} \sin ^{2} \theta}=\frac{\rho}{G} \frac{\partial^{2} u_{\phi}}{\partial t^{2}} .
$$

In the usual way we try to separate the variables by putting:

$u_{\phi}=\Theta(\theta) \cdot R(r) \cdot T(t)$.

Substitution of [22] into [21] leads to:

$$
\begin{aligned}
\frac{1}{R} \frac{d^{2} R}{d r^{2}} & +\frac{2}{r R} \frac{d R}{d r}+\frac{1}{r^{2} \Theta} \frac{d^{2} \Theta}{d \theta^{2}}+\frac{\operatorname{ctg} \theta}{r^{2} \Theta} \frac{d \Theta}{d \theta} \\
& -\frac{1}{r^{2} \sin \theta}=\frac{\rho}{G T} \frac{d^{2} T}{d t^{2}}=-\kappa_{1} . \quad[23
\end{aligned}
$$

From the last eq. of [23] it follows:

$T=A \exp i\left(\frac{\kappa_{1} G}{\rho}\right) t \equiv A \exp i \omega t$,

so that

$\kappa_{1}=\frac{\rho \omega^{2}}{G}=\frac{\omega^{2}}{c^{2}}=\left(\frac{2 \pi}{\lambda}\right)^{2}=k_{e}^{2}$,

where $c$ is the velocity of shear waves in the sample, $\lambda$ the wavelength and $k_{e}$ the wavenumber*).

With the aid of [25] it follows from [23]:

$$
\begin{aligned}
& \frac{r^{2}}{R} \frac{d^{2} R}{d r^{2}}+\frac{2 r}{R} \frac{d R}{d r}+k_{e}^{2} r^{2} \\
& \quad=-\frac{1}{\Theta} \frac{d^{2} \Theta}{d \theta^{2}}-\frac{\operatorname{ctg} \Theta}{\theta} \frac{d \Theta}{d \theta}+\frac{1}{\sin ^{2} \theta}=\kappa_{2} .
\end{aligned}
$$

The last eq. of [26] can be written as

$\frac{d^{2} \Theta}{d \theta^{2}}+\operatorname{ctg} \theta \frac{d \Theta}{d \theta}-\Theta\left(\frac{1}{\sin ^{2} \theta}-\kappa_{2}\right)=0$.

\footnotetext{
*) It should be noticed that since the waves are propagating inwards $c$ is a negative quantity and so is $k_{e}$.
}

Changing the independent variable in [27] from $\theta$ to $x$ by means of $x=\cos \theta$ and $\Theta(\theta)=P(x)$ and writing $\kappa_{2} \equiv v(v+1)$ we get Legendre's differential equation of order 1 :

$$
\begin{aligned}
&\left(1-x^{2}\right) \frac{d^{2} P}{d x^{2}}-2 x \frac{d P}{d x} \\
&+P\left[v(v+1)-\frac{1}{1-x^{2}}\right]=0
\end{aligned}
$$

The solutions of [28] are given by

$$
\left.\begin{array}{l}
P_{v}^{1}(x)=\left(x^{2}-1\right)^{\frac{1}{2}} \frac{d P_{v}(x)}{d x} \\
\text { and } \\
Q_{v}^{1}(x)=\left(x^{2}-1\right)^{\frac{1}{2}} \frac{d Q_{v}(x)}{d x}
\end{array}\right\}
$$

where $P_{v}(x)$ and $Q_{v}(x)$ are associated Legendrefunctions (spherical harmonics) of degree $v$ and order zero and of the first and second kind respectively. When $v=n$, a non-negative integer, $P_{n}(x)=P_{n}(\cos \theta)$ is a Legendre polynomial. It is obvious therefore to consider solutions of [21] which have the form $R_{n}(r) P_{n}^{1}(\cos \theta) \exp i \omega t$, where $R_{n}(r)$ is the solution of the first equation of [26] with $\kappa_{2}=n(n+1)$ and to attempt to build up the desired solution $u_{\phi}$ by forming a series

$u_{\phi}=\exp i \omega t \sum_{n=0}^{\infty} A_{n} R_{n}(r) P_{n}^{1}(\cos \theta)$.

Each term of this series satisfies [21].

For $r=r_{2}$ eq. [30] becomes

$u_{\phi}=\exp i \omega t \sum_{n=0}^{\infty} A_{n} R_{n}\left(r_{2}\right) P_{n}^{1}(\cos \theta)$.

The boundary condition [20] requires:

$\varepsilon r_{2} \sin \theta=\sum_{n=0}^{\infty} A_{n} R_{n}\left(r_{2}\right) P_{n}^{1}(\cos \theta)$.

As $P_{1}^{1}(\cos \theta)=\sin \theta$, a possible solution of [21], satisfying the first equation of [20], is formed by [30] with

$$
\left.\begin{array}{lll}
A_{n}=\varepsilon r_{2} / R_{1}\left(r_{2}\right) & \text { for } & n=1 \\
\text { and } & & \\
A_{n}=0 & \text { for } & n \neq 1
\end{array}\right\}
$$

Since, moreover, the series [30] is unique, the solution of [21] reads:

$u_{\phi}=A_{1} R_{1}(r) \sin \theta \exp i \omega t$. 
For $n=1$ the first equation of [26] takes the form

$\frac{d^{2} R}{d r^{2}}+\frac{2}{r} \frac{d R}{d r}+\left(k_{e}^{2}-\frac{1 \cdot 2}{r^{2}}\right) R=0$.

Changing the independent variable in [34] from $r$ to $z$ by means of $z=k_{e} r$ and $R(r)=Z(z)$ leads to

$\frac{d^{2} Z}{d z^{2}}+\frac{2}{z} \frac{d Z}{d z}+\left(1-\frac{1 \cdot 2}{z^{2}}\right) Z=0$.

Particular solutions of [35] are the spherical
Bessel-functions of the first kind $j_{1}(z)$ and of the second kind $y_{1}(z)$, where

$\left.\begin{array}{l}j_{1}(z)=\sqrt{\frac{1}{2} \pi / z} J_{\frac{1}{2}}(z) \\ \text { and } \\ y_{1}(z)=\sqrt{\frac{1}{2} \pi / z} Y_{\frac{1}{2}}(z)\end{array}\right\}$.

So the solution of [34] is given by

$R(r)=B j_{1}\left(k_{e} r\right)+C y_{1}\left(k_{e} r\right)$.

From [33] and [37] it follows:

$u_{\phi}=\frac{\varepsilon r_{2} \sin \theta\left\{B j_{1}\left(k_{e} r\right)+C y_{1}\left(k_{e} r\right)\right\}}{\left\{B j_{1}\left(k_{e} r_{2}\right)+C y_{1}\left(k_{e} r_{2}\right)\right\}} \exp i \omega t$,

which together with the second equation of [20] finally leads to:

$u_{\phi}=\frac{\varepsilon r_{2} \sin \theta\left\{j_{1}\left(k_{e} r\right) y_{1}\left(k_{e} r_{1}\right)-j_{1}\left(k_{e} r_{1}\right) y_{1}\left(k_{e} r\right)\right\}}{\left\{j_{1}\left(k_{e} r_{2}\right) y_{1}\left(k_{e} r_{1}\right)-j_{1}\left(k_{e} r_{1}\right) y_{1}\left(k_{e} r_{2}\right)\right\}} \exp i \omega t$.

By substituting for $j_{1}\left(k_{e} r_{1}\right)$ etc. the explicit formulae (6), expanding the occurring trigonometric functions and delicate rearranging the terms, it can be shown that the denominator can be written as

$-\frac{\left(r_{2}^{3}-r_{1}^{3}\right)}{3 k_{e} r_{1}^{2} r_{2}^{2}}\left[1-\frac{\left\{k_{e}\left(r_{2}-r_{1}\right)\right\}^{2}}{6}+\frac{\left\{k_{e}\left(r_{2}-r_{1}\right)\right\}^{4}}{120}+0\left\{k_{e}\left(r_{2}-r_{1}\right)\right\}^{5} \ldots\right]$.

The factor in braces of the numerator is found by substituting $r$ for $r_{2}$ in [39]. So for $\left|k_{e}\left(r_{2}-r_{1}\right)\right| \ll 1$ [38] renders into

$u_{\phi}=\varepsilon \gamma \sin \theta\left(r-\frac{r_{1}^{3}}{r^{2}}\right)$

where $\gamma$ is given by [14], eqs. [40] is identical with [15], so we see that in the limit for $\left|k_{1}\left(r_{2}-r\right)\right| \rightarrow 0$ the exact solution [38] renders into [15], where inertia effects have been ignored from the beginning.

For a linear viscoelastic fluid in [38] $k_{e}$ has to be substituted by $k^{*} \equiv-\omega\left(\rho / G^{*}\right)^{\frac{1}{2}}$. In particular for a Newtonian fluid we have:

$k^{*}=k^{\prime}-i k^{\prime \prime}=-\left(\frac{\rho \omega}{2 \eta}\right)^{\frac{1}{2}}(1-i)$,

so

$k^{\prime}=k^{\prime \prime} \equiv k=-\left(\frac{\rho \omega}{2 \eta}\right)^{\frac{1}{2}}$,

where $\eta$ is the viscosity of the fluid.

From [38], [41] and [42] it follows

$u_{\phi}^{*}=u_{\phi}^{\prime}+i u_{\phi}^{\prime \prime}=\frac{\varepsilon r_{2} \sin \theta\left[j_{1}\{k(1-i) r\} y_{1}\left\{k(1-i) r_{1}\right\}-j_{1}\left\{k(1-i) r_{1}\right\} y_{1}\{k(1-i) r\}\right]}{\left[j_{1}\left\{k(1-i) r_{2}\right\} y_{1}\left\{k(1-i) r_{1}\right\}-j_{1}\left\{k(1-i) r_{1}\right\} y_{1}\left\{k(1-i) r_{2}\right\}\right]} \exp i \omega t$.

Taking $\theta=\pi / 2$ and dropping the factor $\exp i \omega t$ in [43], it determines the locus of the centres, i.e. each surface $r=$ constant rotates as if rigid with the angular velocity $\omega$ about the point $u_{\phi}^{\prime} ; u_{\phi}^{\prime \prime}$. Equating the real and imaginary parts of both sides of [43] leads to intractable formulae of $u_{\phi}$ and $u_{\phi}^{\prime \prime}$, however, which will not be given here.

Typical dimensions of the hemispheres used in commercial apparatus are $r_{1}=21 \mathrm{~mm}, r_{2}=22 \mathrm{~mm}$ for the Képès (7) rheometer, and $r_{1}=12.5 \mathrm{~mm}, r_{2}=13.5 \mathrm{~mm}$ for the mechanical spectrometer of 
Rheometrics (8). In fig. 3 a the projections of the locus of rotation centres on the $y-z$ plane and the $x-y$ plane are sketched for the former hemispheres with $\left|k\left(r_{2}-r_{1}\right)\right|=0, \frac{1}{2}, 1$ and $\frac{3}{2}$ respectively.

\subsection{Flow field of an arbitrary linear viscoelastic fluid}

In general we have

$k^{*}=k^{\prime}(1-i \tan \delta / 2)$,

where

$k^{\prime}=\frac{\omega c^{\prime}}{c^{\prime 2}+c^{\prime 2}}=\frac{\omega}{c}=\frac{2 \pi}{\lambda}=-\omega \cos \delta / 2\left(\frac{\rho \cos \delta}{G^{\prime}}\right)^{\frac{1}{2}}$,

with $c$ and $\lambda$ the phase velocity and the wave-length respectively (9) and
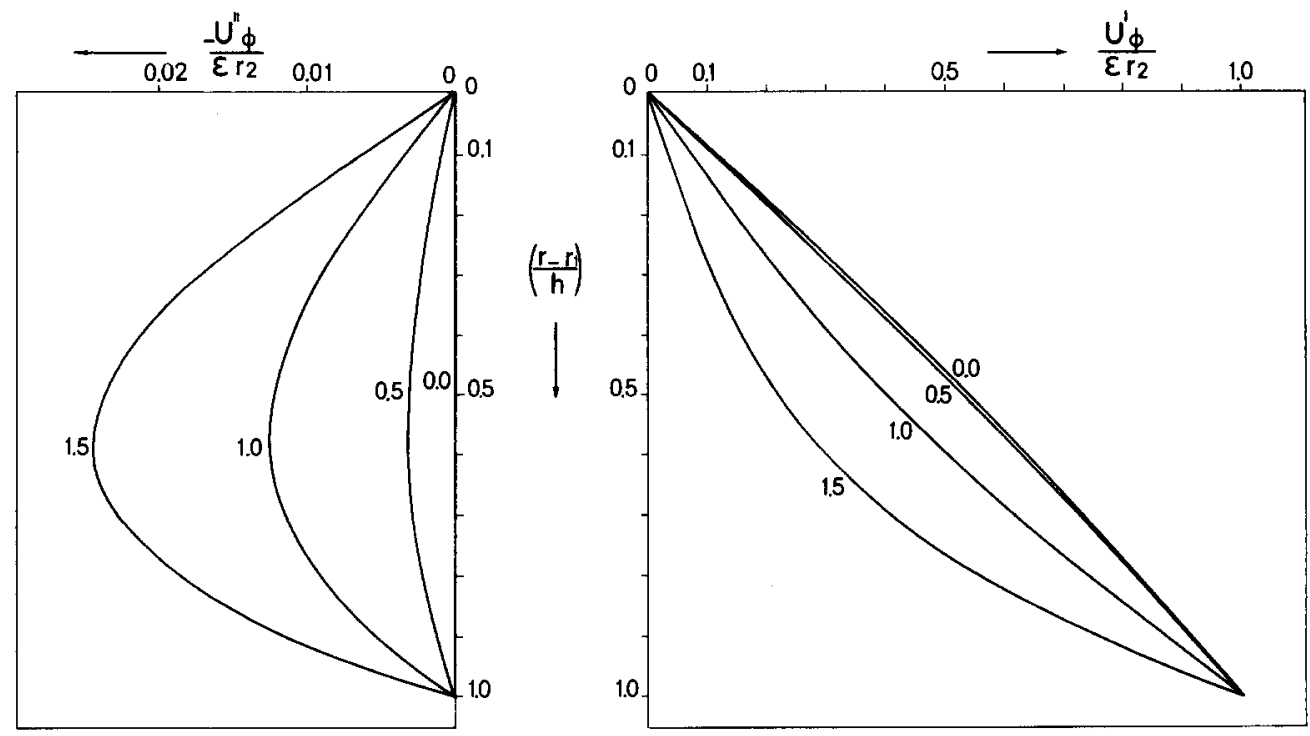

a)
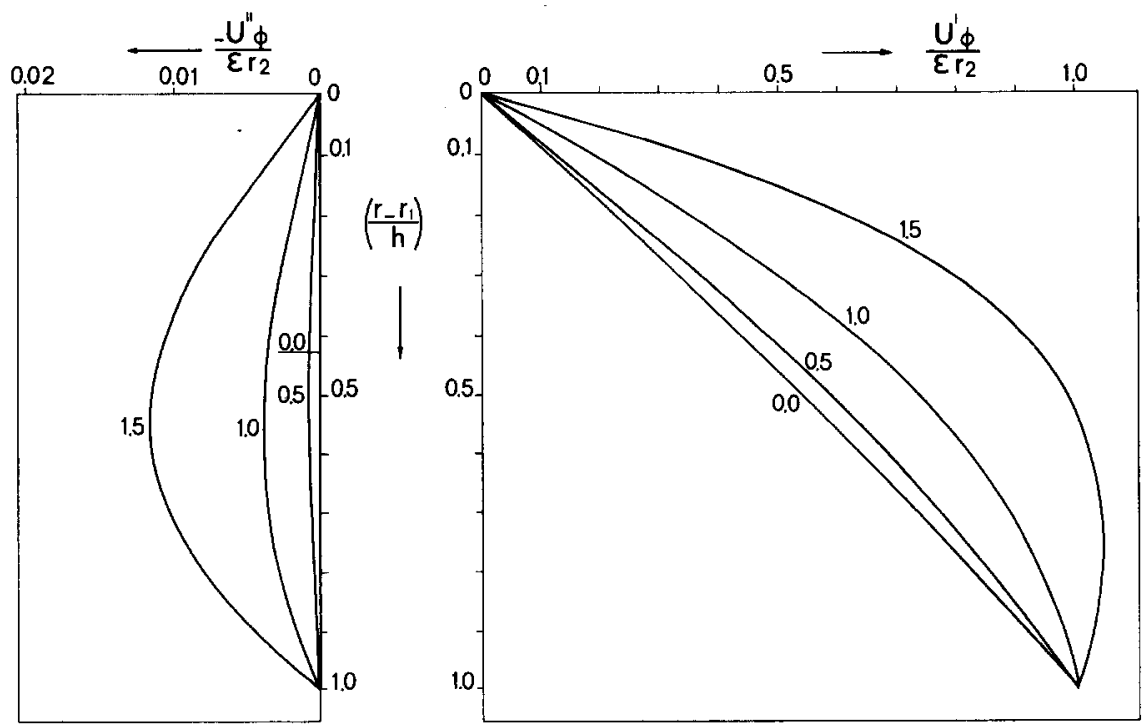

b) 

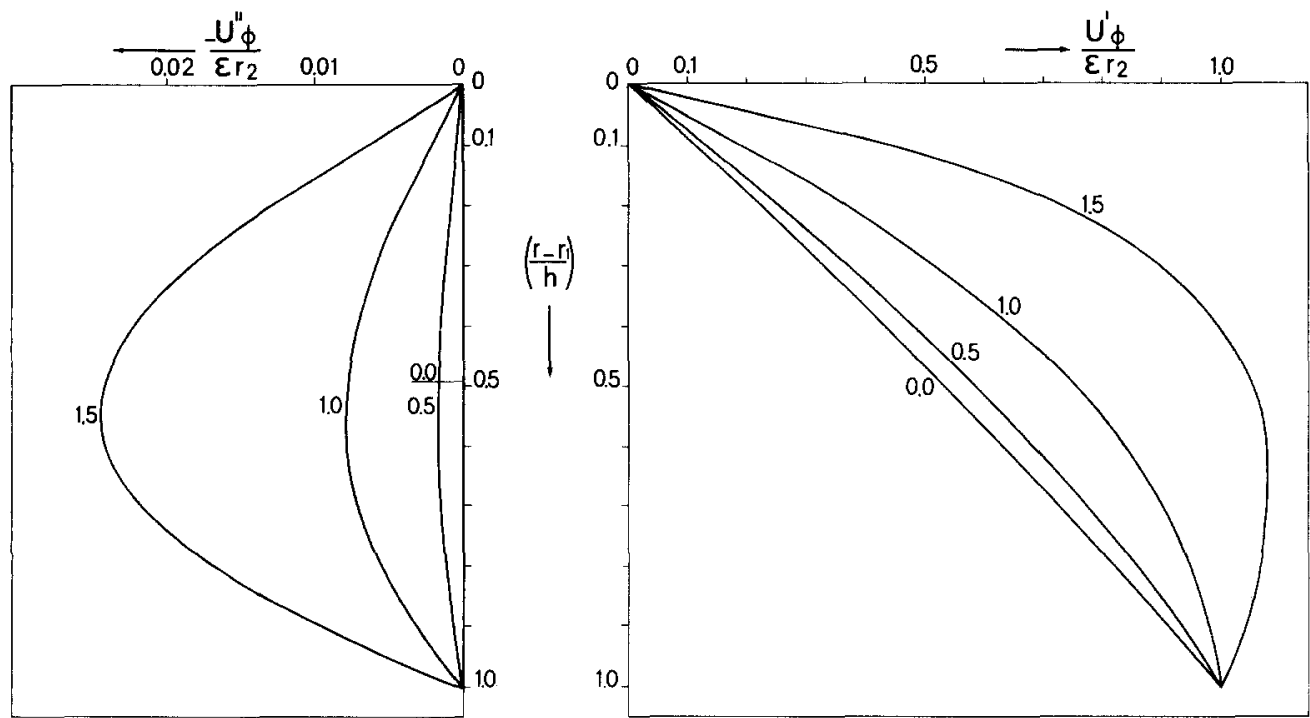

c)
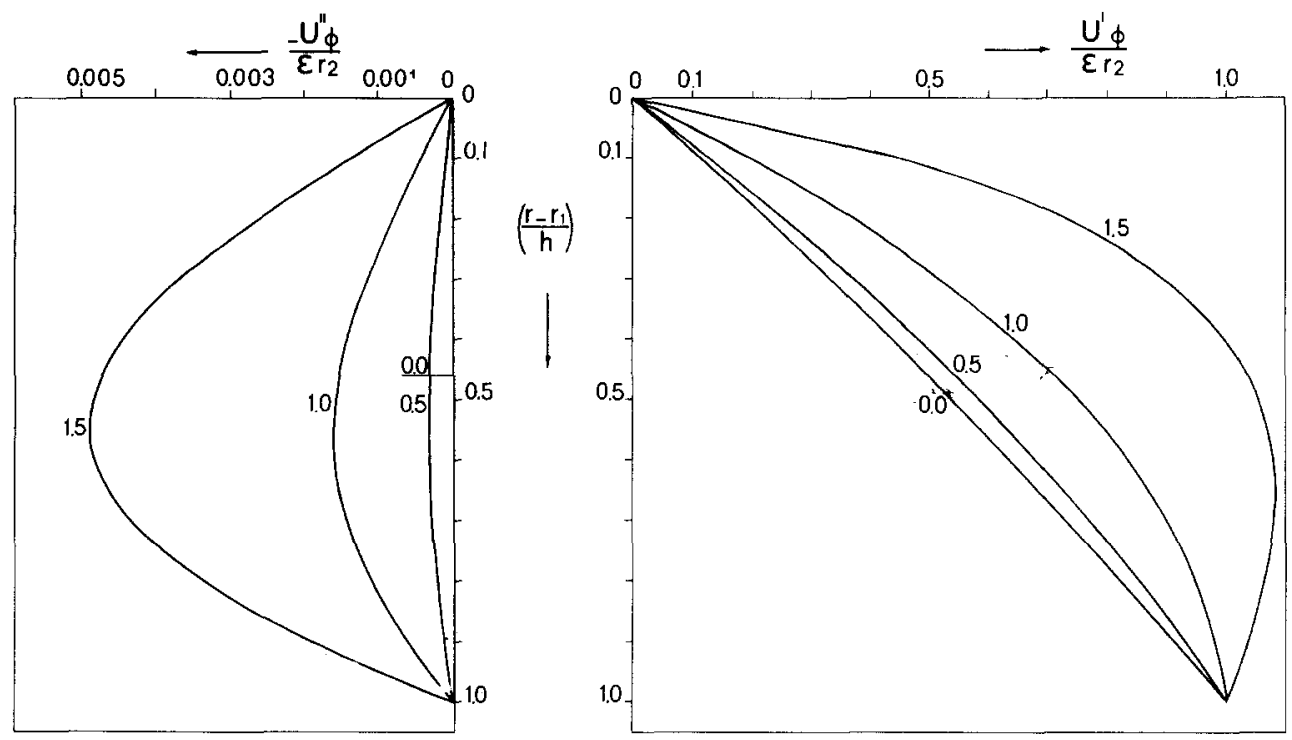

d)

Fig. 3. Projections of the locus of the centres of rotation on the $y-z$ plane and the $x-y$ plane respectively, for $\left|k^{\prime} h\right|=0,1 / 2,1$ and $3 / 2$, see fig. 2 .

a) $\tan \delta / 2=1$; b) $\tan \delta / 2=0.25$; c) $\tan \delta / 2=0.05$; d) $\tan \delta / 2=0.01$

$c^{*}=c^{\prime}+i c^{\prime \prime}=-\left(\frac{G^{*}}{\rho}\right)^{\frac{1}{2}}$

the complex velocity of propagation of the waves.

From [38] and [44] the locus of rotation centres is calculated, see figs. $3 b-3 \mathrm{~d}$.

\section{Forces and couples on the outer sphere}

\subsection{Exact solutions}

As pointed out earlier in this paper the deformation of the sample can be described in a rotating coordinate system as a superposition of two harmonic deformations; one deformation due to a 
harmonic oscillation of the outer sphere about the $z$-axis, the other one due to a harmonic oscillation of the outer sphere about the $y$-axis, see fig. 2. Both oscillations have the same angle amplitude $\varepsilon$, but they show a phase difference of $\pi / 2$ radians. The resulting deformation can be imagined as caused by a harmonic oscillation of the outer sphere about an axis in the $z-x$ plane which turns anticlockwise with the angular velocity $\omega$. As the rotating coordinate system itself turns clockwise with the angular velocity $\omega$, the resulting forces and couples are fixed in space.

For the determination of the forces on the inner sphere it suffices therefore to calculate the (complex) amplitude of the force due to one of the composite harmonic oscillations.

The shear stress in the sample is found from

$\tau_{r \phi}=2 G^{*} S_{r \phi}$,

where

$S_{r \phi}=\frac{1}{2}\left\{\frac{1}{r \sin \theta} \frac{\partial u_{r}}{\partial \phi}+\frac{\partial u_{\phi}}{\partial r}-\frac{u_{\phi}}{r}\right\}$.

From [38] with $k_{e}$ substituted by $k^{*}$ it follows

$\left.\frac{u_{\phi}}{r}\right|_{r=r_{2}}=\varepsilon \sin \theta \exp i \omega t$

and

$\left.\frac{\partial u_{\phi}}{\partial r}\right|_{r=r_{2}}=\varepsilon \sin \theta \exp i \omega t+\frac{k^{*} \varepsilon r_{2} \sin \theta\left\{j_{1}\left(k^{*} r_{1}\right) y_{2}\left(k^{*} r_{2}\right)-j_{2}\left(k^{*} r_{2}\right) y_{1}\left(k^{*} r_{1}\right)\right\}}{\left\{j_{1}\left(k^{*} r_{2}\right) y_{1}\left(k^{*} r_{1}\right)-j_{1}\left(k^{*} r_{1}\right) y_{1}\left(k^{*} r_{2}\right)\right\}} \exp i \omega t$.

So from [46]-[49] we find for the (complex) amplitude $\hat{\tau}_{r \phi}$ of the shear stress:

$\hat{\tau}_{r \phi}=\frac{G^{*} k^{*} \varepsilon r_{2} \sin \theta\left\{j_{1}\left(k^{*} r_{1}\right) y_{2}\left(k^{*} r_{2}\right)-j_{2}\left(k^{*} r_{2}\right) y_{1}\left(k^{*} r_{1}\right)\right\}}{\left\{j_{1}\left(k^{*} r_{2}\right) y_{1}\left(k^{*} r_{1}\right)-j_{1}\left(k^{*} r_{1}\right) y_{1}\left(k^{*} r_{2}\right)\right\}}$.

The force on the outer sphere is found from

$$
\begin{aligned}
F_{x}^{*} & =F_{x}-i F_{z}=r_{2}^{2} \int_{0}^{\pi} \int_{0}^{-\pi} \hat{\tau}_{r \phi} \sin \theta \sin \phi d \theta d \phi \\
& =\frac{\pi G^{*} k^{*} \varepsilon r_{2}^{3}\left\{j_{1}\left(k^{*} r_{1}\right) y_{2}\left(k^{*} r_{2}\right)-j_{2}\left(k^{*} r_{2}\right) y_{1}\left(k^{*} r_{1}\right)\right\}}{\left\{j_{1}\left(k^{*} r_{2}\right) y_{1}\left(k^{*} r_{1}\right)-j_{1}\left(k^{*} r_{1}\right) y_{1}\left(k^{*} r_{2}\right)\right\}} .
\end{aligned}
$$

The same approach given before for the forces is applicable to the couples on the outer hemisphere as well. So the couple on the outer hemisphere is constant in an Eulerian coordinate system and has the magnitude

$$
\begin{aligned}
M_{z}^{*} & =M_{z}+i M_{x}=r_{2}^{3} \int_{0}^{\pi} \int_{0}^{-\pi} \hat{\tau}_{r \phi} \sin ^{2} \theta d \theta d \phi \\
& =\frac{4 \pi G^{*} k^{*} \varepsilon r_{2}^{4}\left\{j_{1}\left(k^{*} r_{1}\right) y_{2}\left(k^{*} r_{2}\right)-j_{2}\left(k^{*} r_{2}\right) y_{1}\left(k^{*} r_{1}\right)\right\}}{3\left\{j_{1}\left(k^{*} r_{2}\right) y_{1}\left(k^{*} r_{1}\right)-j_{1}\left(k^{*} r_{1}\right) y_{1}\left(k^{*} r_{2}\right)\right\}} .
\end{aligned}
$$

In fig. 4 the reduced force on the outer hemisphere

$$
\frac{F_{x}^{*}}{\pi r_{2}^{2}\left|G^{*}\right| \varepsilon r_{2} /\left(r_{2}-r_{1}\right)}=\left(F_{x}\right)_{R}-i\left(F_{z}\right)_{R}
$$

(for the same hemispheres as in fig. 3) is shown for various values of $\left|k^{\prime}\left(r_{2}-r_{1}\right)\right|$ with $\tan \delta / 2$ as a parameter. The values of $\tan \delta / 2$ along the curves are given in the figure. It is seen from this figure that for a constant value of $\tan \delta / 2$ the $F_{x}$-force is a decreasing function of $\left|k^{\prime}\left(r_{2}-r_{1}\right)\right|$. This behaviour is a consequence of resonance phenomena and is most pronounced for small values of $\tan \delta / 2$. In particular for $\tan \delta / 2=0$ it follows from [51] that $F_{x}=0$ for 


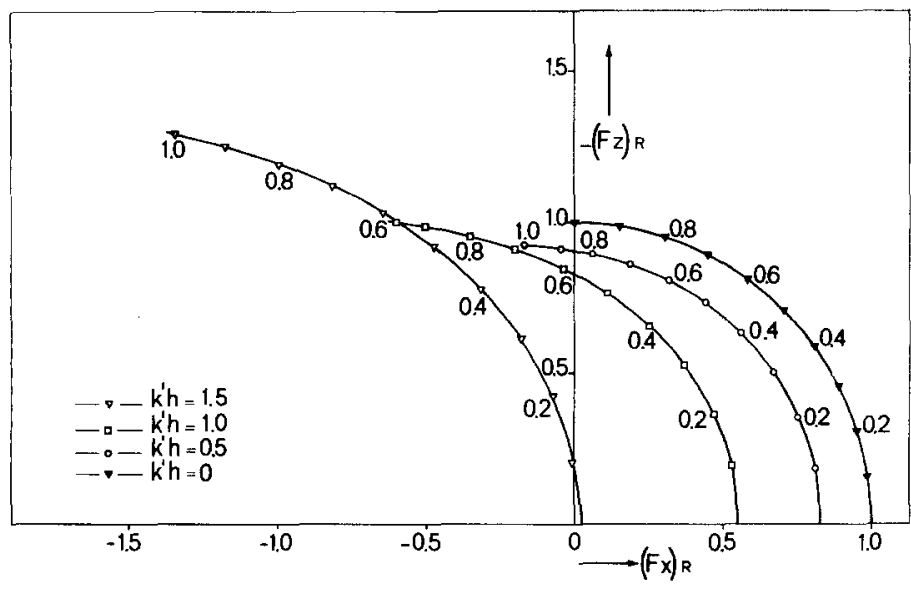

$\left|k^{\prime}\left(r_{2}-r_{1}\right)\right| \equiv\left|k^{\prime} h\right|=\operatorname{arctg}-\frac{k^{\prime 2} r_{1} r_{2}-3 k^{\prime} r_{1}+3}{3 k^{\prime} r_{1}-k^{\prime} r_{2}+3}$,
Fig. 4. Reduced force on the outer hemisphere as a function of $\tan \delta / 2$ and $\left|k^{\prime} h\right|=0,1 / 2,1$ and $3 / 2$ in a polar diagram, see fig. 2

which for the hemispheres considered means: $\left|k^{\prime} h\right|=1.661 ; 4.684$ etc. From fig. 4 it is learned that for a Newtonian fluid $F_{x} \neq 0$. Since $F_{x}$ is proportional to the energy stored in the sample and no elastic energy can be stored in a Newtonian fluid, $F_{x}$ is determined by the stored kinetic energy. Further particulars on this point with respect to the orthogonal rheometer can be found in a previous paper (1), whereas for the balance rheometer it will be given in a forthcoming paper.

\subsection{Working formulae}

Eqs. [51] and [52] are rather inconvenient from the experimenter's point of view. By substituting the explicit formulae for the spherical Bessel-functions in the factor in braces of the numerator of [51] and expanding the occurring trigonometric functions we get:

$$
-\frac{r_{1}}{k^{* 2} r_{2}^{3}}\left[1-\frac{1+2\left(r_{2} / r_{1}\right)^{2}}{3 !}\left(k^{*} h\right)^{2}+\frac{1+4\left(r_{2} / r_{1}\right)^{2}}{5 !}\left(k^{*} h\right)^{4}-\frac{1+6\left(r_{2} / r_{1}\right)^{2}}{7 !}\left(k^{*} h\right)^{6}+\cdots\right] \text {. }
$$

From [51], [39] and [53] it is then found:

$F_{x}^{*}=3 \pi \varepsilon \gamma \frac{r_{1}^{3}}{r_{2}} G^{*}\left[1-\frac{\left(r_{2} / r_{1}\right)^{2}}{3}\left(k^{*} h\right)^{2}-\frac{\left(r_{2} / r_{1}\right)^{2}}{45}\left(k^{*} h\right)^{4}-\frac{2\left(r_{2} / r_{1}\right)^{2}}{945}\left(k^{*} h\right)^{6}-\cdots\right]$.

A similar relation is found for $M_{z}^{*}$.

Expansions of $M_{z}^{*}$ and $F_{x}^{*}$ were given previously by Walters (2) and Bowen (3). They are not in accordance with the expansions given here.

\subsection{The orthogonal rheometer as a limiting case of the balance rheometer}

When both $r_{1}$ and $r_{2}$ goes to infinity but $\varepsilon r_{2}$ is kept constant, $\varepsilon r_{2}=a$, the balance rheometer passes into the orthogonal rheometer. For plates with radius $r_{0}$ it is then found from [50], with $\theta=\pi / 2$ :

$$
\begin{aligned}
F_{x}^{*} & =\pi r_{0}^{2} a G^{*} k^{*} \lim _{\substack{r_{1} \rightarrow \infty \\
r_{2} \rightarrow \infty \\
r_{2}-r_{1}=h}} \frac{\left\{j_{1}\left(k^{*} r_{1}\right) y_{2}\left(k^{*} r_{2}\right)-j_{2}\left(k^{*} r_{2}\right) y_{1}\left(k^{*} r_{1}\right)\right\}}{\left\{j_{1}\left(k^{*} r_{2}\right) y_{1}\left(k^{*} r_{1}\right)-j_{1}\left(k^{*} r_{1}\right) y_{1}\left(k^{*} r_{2}\right)\right\}} \\
& =\pi r_{0}^{2} a G^{*} k^{*} \lim _{\substack{r_{1} \rightarrow \infty \\
r_{2} \rightarrow \infty \\
r_{2} \rightarrow r_{1}=h}} \frac{-\left(k^{* 2} r_{2}^{2}-3 k^{* 2} r_{1} r_{2}-3\right) \sin k^{*} h-\left(k^{* 3} r_{2}^{2} r_{1}+3 k^{*} r_{2}-3 k^{*} r_{1}\right) \cos k^{*} h}{-\left\{\left(1+k^{* 2} r_{1} r_{2}\right) \sin k^{*} h-k^{*} h \cos k^{*} h\right\} k^{*} r_{2}}
\end{aligned}
$$




$$
=\frac{\pi r_{0}^{2} a G^{*} k^{*} \cos k^{*} h}{\sin k^{*} h}=\frac{\pi r_{0}^{2} a G^{*} i k^{*}}{\tanh i k^{*} h} .
$$

Eq. [55] represents the exact expression for the plate forces of the orthogonal rheometer $\left.(1,2)^{*}\right)$. By expanding the hyperbolic function it is found:

$$
F_{x}^{*}=\frac{a \pi r_{0}^{2} G^{*}}{h}\left\{1-\frac{\left(k^{*} h\right)^{2}}{3}-\frac{\left(k^{*} h\right)^{4}}{45}-\cdots\right\} \text {. }
$$

It is worthwile to note that eq. [56] is also found from [54] in the limit for $r_{1} \rightarrow \infty, r_{2} \rightarrow \infty, r_{2}-r_{1}=h$ and $\varepsilon r_{2}=a$, which is not the case for the expansion given by Bowers (3).

\section{Acknowledgements}

I have benefitted from discussions with Dr. F. W. Wiegel. Computer programs for the construction of figs. 3 and 4 were made by $S$. S. Makh (University of Technology, Loughborough) during an industrial training period in our laboratory. Moreover, Mr. Makh derived the series expansions given in eqs. [53] and [54].

\section{Summary}

The flow field of a linear viscoelastic fluid in the balance rheometer, taking fluid inertia into account, has been studied theoretically and an exact solution is given. The flow field of a Newtonian fluid is included in this solution as a special case. The forces and couples on the hemispheres are readily deduced from this solution.

\section{*) Note added in proof}

Actually eq. [55] applies to an experiment with sample thickness $2 h$ and a shift of the plate axis of $2 a$. This discrepancy can be eliminated by changing the boundary conditions [20] in such a way that they become symmetric. Symmetric boundary conditions were used by Abbot and Walters (10) in dealing with the orthogonal rheometer, but not by Walters (2) in the case of the balance rheometer. Contrarily to my prior opinion ((1), see footnote on page 449) I now think that for physical reasons symmetric boundary conditions have to be used in the procedure presented. We will come back to this point in a forthcoming paper. With respect to the present paper symmetric boundary conditions lead to slightly more complicated expressions for forces and couples, while the curves shown in figs. $3 \mathrm{a}$-d will have an " $S$ "-shape just as found for the orthogonal rheometer $(1,2)$.

\section{Zusammenfassung}

Das Strömungsfeld einer linear-viskoelastischen Flüssigkeit im Képès-Rheometer wird unter Berücksichtigung der Flüssigkeitsträgheit theoretisch untersucht und eine exakte Lösung angegeben. Das Strömungsfeld einer newtonschen Fliissigkeit ergibt sich als ein Sonderfall dieser Lösung. Die auf die Halbkugeln ausgeübten Kräfte und Drehmomente lassen sich in einfacher Weise aus der Lösung ableiten.

\section{References}

1) Waterman, H. A., Rheol. Acta 15, 444 (1976).

2) Walters, K., J. Fluid Mech. 40, 191 (1970).

3) Bowen, C. $W$., Phys. D. Thesis, University of Wales (1973).

4) Landau, L. D., E. M. Lifshitz, Fluid Mechanics, Vol. 6 of Course of Theoretical Physics, p. 68 (1963).

5) Abbott, T. N. G., G. W. Bowen, K. Walters, J. Phys. D. 4, 190 (1971).

6) Abramovitz, M., J. A. Stegun, Handbook of Mathematical Functions (New York).

7) Képès, A., unpublished. Manufactured commercially by Contraves, A. G., Switzerland.

8) Rheometrics, Inc., Union. N. J., For a detailed description of this instrument see e.g. Macosko, C.W., Phys. D. Thesis, Dept. Chem. Eng., Princeton University (1970).

9) Waterman, H. A., J. Phys. D. 3, 290 (1970).

10) Abbott, T. N. G., K. Walters, J. Fluid Mech. 40, 205 (1970).

Author's address:

\section{Ir. H. A. Waterman}

Department of Applied Physics

Twente University of Technology

Enschede (The Netherlands) 